\title{
Learning Words through Overhearing
}

Nameera Akhtar, Jennifer Jipson, and Maureen A. Callanan

Recent research indicates that toddlers can monitor others’ conversations, raising the possibility that they can acquire vocabulary in this way. Three studies examined 2-yearolds' $(N=88)$ ability to learn novel words when overhearing these words used by others. Children aged 2,6 were equally good at learning novel words — both object labels and action verbs - when they were overhearers as when they were directly addressed. For younger 2year-olds $(2,1)$, this was true for object labels, but the results were less clear for verbs. The findings demonstrate that 2-year-olds can acquire novel words from overheard speech, and highlight the active role played by toddlers in vocabulary acquisition.

\section{INTRODUCTION}

In Western middle-class communities, young toddlers are sometimes directly taught new words by adults and older children. The rapid rate at which their vocabulary develops, however, suggests that word learning must also occur in less didactic situations (Bloom, 1998). In support of this proposal, recent studies indicate that 2-year-old children can learn new words in a variety of nonostensive contexts, that is, contexts in which an adult's primary intention is not to point out and name an entity for the child and the designated entity is not necessarily perceptually present at the time the word is uttered. In one study, children aged 2,0 were exposed to a new word in the context of a finding game (Akhtar \& Tomasello, 1996). An adult used the novel object label to announce her intention to retrieve an object from a hiding place the contents of which the children were familiar with from previous experience. The children learned the new word even though the referent object was not perceptually present during or after the word's introduction. 
Similarly, 2-year-old children are better able to learn a novel verb when it is used to announce or request an upcoming action than when it is used to label a current, perceptually available action (Tomasello \& Kruger, 1992). Furthermore, a recent study (Callanan, Akhtar, Sussman, \& Sabbagh, 2001) suggests that 24-month-old children can learn a novel object label just as easily when it is used in a directive statement—for example, "Put the toma down here"—as when it is used in an explicit labeling statement—for example, "This is a toma."

The main point of these earlier studies is that 2-year-old children do not learn words solely in the context of adult naming lessons. They are also capable of learning new words in the flow of ongoing social interactions in which an adult uses novel language to regulate behavior, anticipate upcoming events, comment on past experiences, or, in general, "do things with words" other than explicitly teach them to children. These earlier studies, along with many others reviewed in Akhtar and Tomasello (1998) and Akhtar and Tomasello (2000), demonstrate that 2year-olds are actively engaged in determining the referential intentions of adults and can do so in a wide variety of circumstances. What the studies have in common is that they all involve situations in which an adult is directly addressing the child. An interesting question is whether children are able to pick up new words in situations where they are not being directly addressed, that is, situations in which they are overhearers of a third-party conversation.

Adults are quite good at extracting information from overheard speech (Fox Tree, 1999) and several researchers have suggested that children in some parts of the world might have to learn language through this type of "eavesdropping.” In some communities, infants and young children are rarely included as direct addressees in conversational interactions with adults, at least not to the extent that they are in Western middle-class communities (see, e.g., Crago, Allen, \& HoughEyamie, 1997 on an Inuit community in northern Quebec; Brown, 1999 on a Mayan town in highland Chiapas; Schieffelin \& Ochs, 1983 on Papua New Guinea; the review by Lieven, 1994). There is relatively little evidence on the role that siblings and other caregivers play in language so- 
cialization in these communities (but see Schieffelin, 1990; Zukow-Goldring, 1995), but most ethnographic reports suggest that young children certainly experience fewer one-on-one languageteaching interactions than their counterparts in industrialized societies. It is commonly assumed that children in these communities must initially learn some, if not most, of their language by overhearing it used by others.

Indeed, it has been argued that in all cultures the overhearing context is an important one for children who are acquiring language and other social-cognitive skills (Forrester, 1992, 1993). It is well known that children learn a great deal about the rules of their culture by observing the interactions of others. In this article, however, the focus was on whether young children (2-yearolds) could acquire novel words in the overhearing context. To do so, the child must, at the very least, pay close attention to the conversations of others and must be able to segment the target word(s) from overheard speech. There is some evidence suggesting that young children can do both.

First, observational studies indicate that Western, middle-class toddlers monitor third-party conversations. For example, 2- and 3-year-olds often interrupt conversations between their parents and siblings in relevant, topic-sensitive ways (Dunn \& Shatz, 1989). Moreover, research has shown that even younger children (19-month-olds) are more likely to join an ongoing conversation between their mother and older sibling than to initiate one themselves (Barton \& Tomasello, 1991). These toddlers also appropriately respond to requests directed to another person, indicating that they are clearly attending to and comprehending language that is not addressed to them.

Second, somewhat older children (6-year-olds) can extract words from continuous speech even when they have not been instructed to listen to the speech, that is, when the speech is only background "noise” to an unrelated task (Saffran, Newport, Aslin, Tunick, \& Barrueco, 1997). Saffran et al. (p. 104) suggest that such "passive exposure [may be] sufficient for at least some 
aspects of language learning.” These findings demonstrate that children can perform word segmentation "incidentally," without paying active attention to the speech stream and without the benefit of familiar words in the acoustic stimuli they were exposed to. Given that there is abundant motivation for younger children to attend to the conversations of their siblings and parents and that they are already familiar with some of the language they are overhearing (Goodman, McDonough, \& Brown, 1998), it is quite plausible that they might also be able to extract and learn novel words from speech they overhear.

Recent work by Oshima-Takane $(1988,1999)$ suggests that overhearing may play a particularly important role in the acquisition of personal pronouns. Some children make reversal errors with personal pronouns, for example, referring to themselves as "you." One possible reason for these errors is that personal pronouns, along with other deictic terms, have a shifting reference-that is, the person referred to by a particular pronoun changes with regard to who is the speaker and who is the addressee. When a mother speaks to her child, for instance, she refers to herself as "I" or "me" and the child as "you," but when the child speaks, the usage of the pronouns must be reversed. Consequently, the correct usage of personal pronouns depends on understanding the relations between the pronouns and speech roles. As Oshima-Takane (1988) points out, however, models for correct usage are not available in child-directed speech alone. For example, if children only hear the word "you" in reference to themselves, they might reasonably conclude that "you" is a name for them. But (over)hearing others addressing one another as "you" would lead to the more appropriate inference that "you" is a word used to refer to whomever is being addressed.

In this regard, it is particularly interesting that second born children tend to have an advantage over firstborn children in learning personal pronouns (Oshima-Takane, Goodz, \& Derevensky, 1996). Although their overall rate of language development is equivalent to that of firstborn children, second born children are more likely to correctly use personal pronouns. Moreover, an examination of maternal speech in the triadic context (mother, firstborn, and second-born) revealed 
that secondborn children were exposed to more pronouns in overheard conversations than in speech directed to them. Oshima-Takane et al. (1996) interpret these findings as support for the hypothesis that second born children's advantage in pronoun production is linked to their opportunities to overhear pronouns being used in third-party conversations between their siblings and parents.

The literature reviewed above establishes that toddlers can and do monitor the conversations of their siblings and parents and suggests that toddlers can take advantage of overheard speech to learn personal pronouns. Anecdotally, many parents report that their children pick up words—often colloquial terms parents find undesirable—overheard in adult speech. To our knowledge, however, no one has systematically investigated children's ability to learn referential terms through overhearing. In addition, as it is known that young, English-speaking children tend to learn object labels more easily than verbs (Gentner, 1982), it is worthwhile to examine how well children learn different types of words though overhearing.

The studies reported in this article directly examined whether young toddlers can learn novel words (object labels and action verbs) by overhearing them used by others. In each study, children were randomly assigned to one of two conditions: Addressed

or Overhearing. In the Addressed condition, the experimenter played a game with the child and introduced a novel word for one of four unfamiliar objects / actions. In the Overhearing condition, the child was positioned as an onlooker to an identical interaction between the experimenter and an adult assistant. In this condition, the experimenter introduced the novel word to the assistant instead of to the child, essentially ignoring the child. The first set of studies (1A and 1B) examined the word-learning abilities of children, age 2,6, in these two situations. The remaining studies (2A, 2B, and 3) examined younger 2-yearolds' performance in the same situations. 


\section{STUDY 1A: OBJECT LABELS Method}

\section{Participants}

Twenty-four children (9 firstborn females, 9 firstborn males, 4 laterborn females, and 2 laterborn

males) participated in this study. They ranged in age from 2,4 to 2,8, $M=2,6, S D=0,1.4$. Participants were identified from a database of children whose parents had expressed interest in being included in studies of child development. The majority of the children were from middle-income, European American backgrounds, and all were native English speakers. Testing was conducted in a laboratory playroom and each child received a small gift for participating. (In all the studies reported, no effects of birth order or gender were found. Note, however, that the cell sizes were not equal, in part because parents of firstborns were overrepresented in our sample of parent volunteers.)

\section{Materials}

Four novel objects were used: a wallpaper roller, a noisemaker, an unusually shaped yo-yo, and a small wooden toy made of two connected disks that wobbled when rolled. Other novel objects (e.g., an oddly shaped spinning top) were available as replacements if a parent reported that the child was familiar with one of the novel objects or if a given child produced a name for any of the four preselected toys. A set of familiar objects (e.g., a small Barney figure, a plastic spoon, a ball, a toy car) were also used in a warm-up comprehension task (see Procedure section). In addition, a hiding apparatus consisting of four buckets mounted in a row on a wooden plank was used in a finding game. When lids were placed on the buckets, the toys inside were not visible.

A star-shaped foam pad was placed on the floor to help participants remember where they were supposed to sit. In the Addressed condition, the foam pad was placed opposite the experimenter (on the other side of the hiding apparatus), less than a foot away. In the Overhearing condition, the 
foam pad was placed a few feet to the left of the experimenter, where children could easily view the experimenter, assistant, and hiding apparatus.

\section{Design}

Children were randomly assigned to one of two conditions: Addressed or Overhearing. In the Addressed condition, the child sat on the floor facing the experimenter with the hiding apparatus between them. The experimenter played a finding game with the child and introduced a novel object label for one of the four unfamiliar objects (see Training rounds section). In the Overhearing condition, an adult assistant sat on the floor facing the experimenter with the hiding apparatus between them. The child was positioned as an onlooker to an interaction between the experimenter and the assistant; during this interaction, the experimenter introduced the novel object label to the assistant in the manner described for the Addressed condition. Each of the four novel toys served as the target object three times in each of the two conditions.

\section{Procedure}

All sessions were conducted by the same female experimenter in a psychology laboratory playroom, and were videotaped by an observer behind a two-way mirror. Each child participated individually in a single half-hour visit. Upon arrival, the parents were asked to examine the novel toys and determine whether these toys were likely to be unfamiliar (i.e., nameless) to their child. If parents reported that their child might be familiar with one or more of the toys, these toys were replaced by others from the replacement set. Parents were also asked to complete a productive vocabulary checklist (Form A of the short version of the MacArthur Communicative Development Inventory; Reznick \& Goldsmith, 1989). The children’s vocabulary scores are shown in Table 1. Random assignment appears to have been successful; the mean vocabulary scores of the children in the two conditions did not differ significantly from one another in any of the studies. 
[Insert Table 1]

After a short interval during which the experimenter played with the child to familiarize him or her with the setting, the experimental session began. Each session was videotaped by the hidden observer, who kept a written record of the child's use of the novel word throughout the session and also recorded which toy was chosen during the comprehension and preference trials (see related section).

Warm-up round. Each child in the Addressed condition first engaged in a warm-up event in which the experimenter opened each bucket one at a time saying, "Let's see what's in this one.” The experimenter then removed the object hidden inside and demonstrated how the object worked. The child was allowed to play with each toy and was asked to replace it before being shown the next one. The purpose of the warm-up round was to familiarize the child with the contents of each bucket, as well as the "rules" of the finding game (e.g., the experimenter must open the buckets, objects must be replaced before the next bucket is opened, and so forth). In the Overhearing condition, the child was told to sit on the star and wait to play while the assistant had a turn first. From this point on, no eye contact was made with the child until it was the child's turn to play. The experimenter then proceeded through the warm-up process with the assistant, with the child as an onlooker.

Training rounds. Following the warm-up round, each child in the Addressed condition, as well as the assistant in the Overhearing condition, played three rounds of the same finding game with the experimenter. Each round consisted of the experimenter finding and revealing each of the four toys (one target and three nontargets) one at a time. Each toy was always found in the same location and in the same order on each round. Finding each of the three nontarget objects was preceded by a series of three neutral utterances (e.g., "I’m going to show you what's in here.” 
“Let's see what's in here.” "I'll show you this one.”). The experimenter then opened the bucket, removed and held up the object found inside, smiled and gasped, and then handed the object to the child (or assistant). Each toy was replaced before the next toy was found. Finding of the target object, however, was preceded by the experimenter introducing the word toma once in each of a series of three utterances (e.g., “I’m going to show you the toma.” “Let's find the toma.” "I'll show you the toma.”). All language models were introduced before the target object became visible, and the target object continued to be found in the same manner described for nontarget objects. Each child heard a total of nine tokens of the word toma: three tokens in each round of the finding game.

After the training period was complete, each child in the Overhearing condition was invited to play with the objects with the experimenter while the assistant moved aside. This game was essentially one round of finding the objects with only neutral language used; that is, the same language that was used for the non-target objects was used for all four objects. After the child had this opportunity to play with each of the objects, the experimenter moved on to the comprehension and preference trials. In the Addressed condition, the experimenter continued directly to the comprehension and preference trials. This procedure led to a somewhat longer delay between the language models and the comprehension and preference trials in the Overhearing condition, but this was necessary to allow each child ample opportunity to handle the objects before proceeding to the comprehension and preference trials.

Familiar-items comprehension. At this point, the hiding apparatus was removed from the child's sight and an aide (or the assistant) quickly removed the toys while the experimenter engaged the child in the comprehension task with familiar items. This task involved asking the child to show or hand to the experimenter objects with known labels (see Materials section) and was designed to elicit giving and showing responses from the child. Four familiar objects were presented on a tray and the experimenter asked the child to identify and select one (e.g., “Can you 
give me the spoon?”). This object was replaced before proceeding to the next trial. After the child had correctly chosen two objects in a row, indicating that he or she had understood and complied with the experimenter's instructions, the experimenter proceeded to the comprehension and preference trials.

[Insert Table 2]

Comprehension and preference trials. In this task, the familiar objects were replaced with the target and nontarget objects from the training rounds, in random positions on the tray, and the child was asked to show or give the toma to the experimenter. The experimenter established eye contact with the child before making this request and did not look at the objects when asking for the toma. If the child did not respond or asked whether a particular object was the toma, the question was repeated until a toy was chosen. To ensure that children were choosing what they believed to be the toma and not just selecting their favorite object, a preference control trial was also included. During the control trial, the target and nontarget objects were placed on the tray and children were asked to show or give the experimenter the "one they liked the best" or their "favorite one." Order of preference trials and comprehension trials was counterbalanced with the experimenter distracting the children (e.g., by commenting on their clothing or something in the room) for 1 to 2 min between each trial.

Reliability coding. The observer behind the two-way mirror noted which object the child chose during each trial. An independent coder then reviewed 25\% of the videotaped sessions (the comprehension and preference trials of three children randomly chosen from each condition); agreement with the original observer was $100 \%$ on these trials. A third observer coded (from videotape) the length of time between the experimenter's last phrase containing the novel label 
and the comprehension trial for each child in each condition. These data are shown in Table 2. The delay was significantly longer in the Overhearing condition in each study, all ps $<.05$.

\section{Results and Discussion}

The number of children $(N=12)$ in each condition who chose the target object in response to the comprehension and preference questions is shown in the first two columns of Table 3. Abovechance performance, $p<.05$, as measured by a binomial test (with chance probability $=.25$ ), required at least 7 of the 12 children to choose the correct item. As shown in Table 3, the number of children who chose the target object during the comprehension trial was greater than chance in both conditions, Addressed and Overhearing. In contrast, the number of children who chose the target in response to the preference question was not greater than that expected by chance. Children performed equally well, $p>.05$ (determined by the Fisher exact probability test), in the Addressed and Overhearing conditions.

Although these results supported the hypothesis that children age 2,6 can learn a novel object label equally well when overhearing it as when being directly addressed, it was necessary to more closely examine the individual patterns of data and take advantage of the within-subjects design employed in this study. That is, it was important to take into account the fact that some of the children may have chosen the target object during both the comprehension and preference trials. Indeed, one child in the Addressed condition (none in the Overhearing condition) did so. When this child's score was subtracted from the comprehension data, the results remained the same: In both conditions, children were more likely than chance to choose the target object during the comprehension trials and as likely as that expected by chance during the preference trials, and there was no significant difference between the two conditions. Sign tests that take into account all three possible combinations of scores (ties, chose target during preference trial but not comprehension trial, chose target during comprehension trial but not preference trial) revealed that a significant 
number of children chose the target object during comprehension trial but not the preference trial in both conditions, $p<.03, p<.01$ for the Addressed and Overhearing conditions, respectively.

The findings of this study clearly demonstrate that, under the current conditions, children age 2,6 are equally good at learning a novel object label when they overhear it as when they are directly addressed. The equivalence of the two conditions is all the more remarkable given that there was a greater delay between hearing the word and being tested for comprehension in the Overhearing condition than in the Addressed condition. Object labels, however, are only one type of word that young children overhear. Study 1B assessed the ability of children, age 2,6, to learn a novel verb through overhearing.

\section{STUDY 1B: ACTION VERBS Method}

\section{Participants}

The same 24 children who participated in Study 1A also participated in this study. The order of studies was counterbalanced. Children's assignment to the two conditions, Addressed or Overhearing, was counterbalanced so that equal numbers were in each of the following groups: Addressed for both noun and verb, Overhearing for both noun and verb, Addressed for noun and Overhearing for verb, Addressed for verb and Overhearing for noun. The design of Study 1B was analogous to that of Study 1A.

\section{Materials}

A set of four toy characters (Elmo, Big Bird, Winnie the Pooh, and Tigger) and four props were used to perform the four novel actions (one target and three nontarget). These props and their associated actions were (1) a tape measure that was made to pull in a ring in which a character sat, (2) a curved platform that catapulted a character into the air when hit on one side, (3) a curved chute through which a character was dropped, and (4) a platform attached to a base by a 
spring that toppled a character off the platform when the spring was bumped. Each character was always paired with the same action. Each of the novel actions served as the target action for three children in each condition. The characters and props were kept hidden in a canvas bag and were extracted one at a time when the actions were demonstrated. A fifth toy character (Cookie Monster) was used for the comprehension and preference trials (see related section). The same star-shaped foam pad from Study 1A was set on the floor to help the children remember where they were supposed to sit.

\section{Design and Procedure}

Warm-up round. Each child in the Addressed condition (and the assistant in the Overhearing condition) first engaged in a warm-up event in which the experimenter individually demonstrated each action, first saying, “I’m going to show you what I can do with [character's name].” In the Overhearing condition, the child was told to sit on the star and wait to play while the assistant had a turn first. From this point on, no eye contact was made with the child until it was his or her turn to play. The experimenter then removed one action prop and its associated character from the canvas bag and demonstrated how the action worked. The child (or assistant) was allowed to try each action before being shown the next one. The purpose of the warm-up exercise was to familiarize each child with each action and the turn-taking procedure.

Training rounds. Following the warm-up exercise, each child in the Addressed condition (and the assistant in the Overhearing condition) played three rounds of a game with the experimenter in which each action was demonstrated. Each round consisted of the experimenter showing each action (one target and three nontargets) one time. Actions were always demonstrated in the same order on each round. Demonstration of each of the three nontarget actions was preceded by three neutral utterances such as "We can do this game with [character's name]." "Let’s try this with [character's name].” "I’ll show you this game.” The 
experimenter then demonstrated the action, smiled and gasped, and then handed the character to the child (or assistant) to perform the action. Each action prop and character was set aside before the next action was demonstrated. Demonstration of the target action was preceded by the experimenter introducing the verb meek three times in a series of utterances such as “Now I’m going to meek [character's name].” “Let’s meek [character's name].” “I’ll show you how to meek [character's name].” All language models were introduced before the action was demonstrated. Therefore, each child heard a total of nine tokens of the verb meek, three tokens in each round of the game.

Comprehension and preference trials. After the training period was complete, each child in the Overhearing condition was invited to play with the experimenter, and the assistant moved aside. The child was then given the opportunity to play with each of the action props and their associated characters. In the Addressed condition, the experimenter and child continued on with the procedure. To show the child that the actions were applicable to characters other than those used in the training rounds, the experimenter took out a different character (Cookie Monster) and demonstrated each action (in random order) with this character. The child was then asked to meek Cookie Monster. The experimenter attempted to establish eye contact with the child before making this request, and did not look at the action props when asking the child to demonstrate the target action.

To ensure that children were demonstrating what they believed to be the target action and not just performing their favorite action, a preference control trial was also included. During this trial, children were asked to show the experimenter the game they liked most to play with Cookie Monster. Order of preference trials and comprehension trials was counterbalanced with the experimenter momentarily distracting the children between each trial (e.g., by commenting on their clothing or something in the playroom).

Reliability coding. The observer behind the two-way mirror made note of which action the child performed during each trial. An independent coder then reviewed 25\% of the videotaped 
sessions (the comprehension and preference trials of three children randomly chosen from each condition); agreement with the original coder was $100 \%$ on these trials.

\section{Results and Discussion}

The number of children $(N=12)$ in each condition who performed the target action in response to the comprehension and preference questions is shown in the last two columns of Table 3. Abovechance performance, $p<.05$, as measured by a binomial test (with chance probability $=.25$ ), required at least 7 of the 12 children to perform the correct action. As shown in Table 3, the number of children who performed the target action during the comprehension trial was greater than chance in both conditions, Addressed and Overhearing. Moreover, children performed equally well, $p>.05$ (determined by the Fisher exact probability test), in the Addressed and Overhearing conditions. In contrast, the number of children performing the target action during the preference trial was not greater than that expected by chance. Only one child in the Addressed condition (none in the Overhearing condition) performed the target action during both the comprehension and preference trials. Sign tests revealed that a significant number of children performed the target action during the comprehension trial but not the preference trial in both conditions, $p<.02$ for both trials.

The findings of this study clearly demonstrate that under the above conditions, children age 2,6 are equally good at learning a novel verb when they overhear it as when they are directly addressed. The equivalence of the two conditions is quite striking given there was a greater delay in the Overhearing condition than in the Addressed condition between hearing the word and being tested for comprehension, and that children did not have to attend to the experimenter and assistant if they chose not to. Together, the results of Studies $1 \mathrm{~A}$ and 1B demonstrate that children, age 2,6, are equally good at learning novel object labels and verbs when they overhear a word as when they are directly addressed. It is not clear, however, whether children younger than age 2,6 are 
also adept at learning words by overhearing them used by others. The remaining studies assessed younger 2-year-olds' ability to learn novel words through overhearing.

\section{STUDY 2A: OBJECT LABELS Method}

\section{Participants}

Thirty-two children (12 firstborn females, 8 firstborn males, 4 later born females, and 8 later born males) participated in this study. Participants ranged in age from 1,11 to 2,2 ( $M=2,1, S D=$ 0,0.9), and were randomly assigned to one of the two conditions. Participants were identified from the same database of children as in Study 1.

\section{Materials}

The materials were the same as those used in Study 1A.

\section{Design and Procedure}

The design and procedure were identical to those of Study 1A. An independent coder reviewed $25 \%$ of the videotaped sessions (four participants' comprehension and preference trials randomly chosen from each condition); agreement with the original coder was $100 \%$ on these trials.

\section{Results and Discussion}

The first column of Table 4 shows the number of children $(N=16)$ in each condition who chose the target object when asked to find the toma. Also shown is the number of children who chose the target object when asked to choose the one they liked best. Above-chance performance, $p<.05$, as measured by a binomial test (with chance probability $=.25$ ), required at least 7 of the 16 children to choose the correct item. As shown in Table 4, the number of children who chose the target object during the comprehension trial was greater than chance in both conditions, Addressed and Overhearing. Moreover, children performed equally well, $p>.05$ (determined by the Fisher exact 
probability test), in the Addressed and Overhearing conditions. The number of children who chose the target object during the preference trial, however, was not greater than chance in either condition.

Three children in the Addressed condition and one in the Overhearing condition chose the target object on both trials. Results of sign tests demonstrated that, even when the data for these children are excluded, the patterns of performance in both conditions were still significantly different from chance, $p<.02$ and $p<.01$ for the Addressed and Overhearing conditions, respectively; more children chose the target object during the comprehension trial and not during the preference trial than would be expected on the basis of chance alone.

Even with the extra memory and attention demands of the Overhearing condition, the younger 2-year-olds were able to learn a new word through overhearing. Indeed, as with the older group of 2-year-olds, these children were able to learn a new object label equally well when it was introduced directly to them as when they overheard its use in a conversation between others. Study 2B tested whether younger 2-year-olds are also able to learn new action verbs through overhearing.

\section{STUDY 2B: ACTION VERBS Method}

\section{Participants}

The same 32 children who participated in Study 2A also participated in Study 2B. The order of studies was counterbalanced. Children's assignment to the two conditions, Addressed or Overhearing, was counterbalanced so that equal numbers were in each of the following groups: Addressed for both noun and verb, Overhearing for both noun and verb, Addressed for noun and Overhearing for verb, Addressed for verb and Overhearing for noun.

Materials 
The materials were the same as those used in Study $1 \mathrm{~B}$.

Design and Procedure

The design and procedure were identical to those of Study 1B. An independent coder reviewed 25\% of the videotaped sessions (four participants' comprehension and preference trials randomly chosen from each condition); agreement with the original coder was $100 \%$ on these trials.

\section{Results and Discussion}

The third column of Table 4 shows the number of children $(N=16)$ in each of the two conditions who performed the target action when asked to meek Cookie Monster. The number of children who performed the target action when asked to play their favorite game with Cookie Monster is given in the final column of Table 4 . Above-chance performance, $p<.05$, as measured by a binomial test (with chance probability $=.25$ ), required at least 7 of the 16 children to perform the target action. As shown in Table 4, the number of children performing the target action during the comprehension trial was greater than chance only in the Addressed condition. The number of children performing the target action during the preference trial was not greater than chance in either condition. This analysis suggests that younger 2-year-olds learn novel verbs only when directly addressed.

[Insert Table 4]

Three children in the Addressed condition and one in the Overhearing condition performed the target action during both the comprehension and preference trials. Therefore, sign tests were conducted to assess performance in each condition. The analyses revealed chance performance in both conditions, $p=.22$ and $p=.62$ in the Addressed and Overhearing conditions, respectively. Thus, it appears that by this stricter measure of learning, the younger 2-year-olds were not able to 
demonstrate that they had learned an action verb in either of the two conditions in this setting. The fact that these children were unable to learn a novel action verb by being directly addressed is at odds with previous literature (see, e.g., Akhtar \& Tomasello, 1996); this discrepancy is considered in detail in the "Results and Discussion" section of Study 3.

Clearly it was harder for the younger 2-year-olds to learn a novel action verb than it was to learn a novel object label. This finding is consistent with claims that verbs are inherently more complex (and therefore more difficult to acquire) than object labels (Gentner, 1978, 1982). A number of possible reasons for this difference are explored in the "General Discussion" section. Study 3 examines one aspect of the current procedure that may have contributed to children's difficulty with verbs in this particular setting. Learning a novel verb may have been difficult for younger 2-year-old children in this study because of the amount of time they had to explore and perform the novel actions before they heard the verb used to describe the target action. Children in the Overhearing condition heard the novel action verb used before they had had a chance to play with the action props, whereas the children in the Addressed condition heard the novel action verb only after first playing with each of the action props. It is possible that children in the Overhearing condition were so focused on the actions themselves (and the fact that they had to wait patiently) while observing the experimenter and assistant playing, that they did not have the attentional resources to focus on the language used by the experimenter. It was thought that providing children with some experience with the actions before introducing the novel verb might boost their performance in this condition. Study 3 was designed to assess this hypothesis.

\section{STUDY 3: ACTION VERBS Method}

\section{Participants}


Thirty-two children (12 firstborn females, 9 firstborn males, 6 laterborn females, 5 laterborn males) participated in this study. They ranged in age from 1,11 to $2,3(M=2,1, S D=0,1.0)$. Participants were identified from the same database of children as in the previous studies; there was no overlap among the children of Studies 2 and 3.

\section{Materials}

The materials were the same as those used in Studies 1B and 2B.

\section{Design and Procedure}

The design was the same as those of the previous studies. There was, however, one significant change in the procedures: In the Overhearing condition, children underwent an initial warm-up round in which they were allowed to play with each of the novel action props before the experimenter began playing with the assistant. This change was designed to allow these children to "get out of their system" the desire to play with the props, and to focus more on the language used by the experimenter.

Once again, an independent coder reviewed 25\% of the sessions (four children’s comprehension and preference trials randomly chosen from each condition); agreement with the original coder was $100 \%$ on these trials.

\section{Results and Discussion}

The first column of Table 5 shows the number of participants $(N=16)$ in each of the two conditions who performed the target action when asked to meek Cookie Monster. The number of children who performed the target action when asked to play their favorite game with Cookie Monster is given in the second column of Table 5. Although comprehension performance was greater than chance in both conditions, the number of children performing the target action in response to the preference question was not greater than chance in either condition. These results 
would seem to suggest that in contrast to the children in Study 2B, younger 2-year-olds presented with the revised procedure were able to demonstrate verb learning in both the Addressed and Overhearing conditions.

\section{[Insert Table 5]}

This conclusion, however, needs to be considered in light of the results of sign tests performed. Because two children in the Addressed condition and one child in the Overhearing condition performed the target action during both the comprehension and preference trials, sign tests were conducted to assess performance in each condition. The results indicated that performance in neither condition differed significantly from chance, $p=.29$ and $p=.12$ in the Addressed and Overhearing conditions, respectively. Thus, using the stricter criterion of not giving children credit for learning if they also performed the target action on the preference trial, it appears that the younger 2-year-olds in this study were not able to clearly demonstrate that they had learned an action verb in either the Addressed or Overhearing conditions.

In attempting to make sense of the differing results obtained by the two statistical comparisons, it is useful to consider these results in light of previous findings. First, there is a discrepancy between the current finding for Addressed children and previous research results. For example, in a study employing a very similar procedure, Akhtar and Tomasello (1996) found that children of this age $(2,0)$ were able to learn novel action verbs when directly addressed. One potentially important procedural difference is that Akhtar and Tomasello used a different type of control than that used in the current studies; that is, instead of the same children undergoing both preference and comprehension trials (as in the current studies), a separate group of children experienced the same procedures as the experimental group but never heard a novel word until the testing phase. Their performance was then compared with that of the experimental group's comprehension 
performance. In the current study, the decision to use a within-subjects design to assess children's comprehension and preference choices was intended to control for the possibility that children may simply select their favorite action in the comprehension task. Although this interpretation may be one way to explain cases in which a child chooses the same action on both trials, there are alternative explanations for this pattern of results. In particular, it is possible that learning a novel verb for an action makes that action more attractive to children. According to this hypothesis, children may be somewhat more likely to select the target action on the preference trial simply because they know the name for that action.

Using the more lenient criterion of comparing performance to chance, however, children in the Overhearing condition were able to demonstrate verb learning in Study 3 but not in Study 2B. This finding leads us to consider the possibility that younger 2- year-olds may be capable of learning action verbs by overhearing them used by others only when other aspects of the wordlearning context are favorable. Study 3 specifically focused on whether children's learning of a novel action verb was influenced by the amount of experience they had with the action prior to hearing the verb used to describe it (Akhtar \& Tomasello, 1996). In comparison to Study 2B, children in the Overhearing condition in Study 3 were allowed greater hands-on experience with the novel actions before being presented with the novel verb. This hands-on experience may have slightly reduced the extent to which the novel actions themselves diverted the children's attention away from the presentation of the novel verb. As support for this hypothesis, it is important to note that the participants in Akhtar and Tomasello's study had much more experience with the novel actions prior to the introduction of any language models. In a sense then, the actions themselves were not quite so novel and attention grabbing by the time the children heard the novel verb; this enabled the children to focus on the word itself and its referent.

\section{GENERAL DISCUSSION}


The studies presented in this article provide evidence regarding young children's abilities to learn novel words when overhearing them used in third-party conversations. The results of our first set of studies demonstrated quite clearly that by age 2,6, children are capable of learning novel words through overhearing. In Study 1A, children, age 2,6, easily learned a novel object label when overhearing it used by others. In Study 1B, the same children successfully learned a novel verb through overhearing. Indeed, children in these studies were equally good at learning novel

words (both object labels and action verbs) when they overheard the word used by others as when they were directly addressed.

That the word learning of children this age was equivalent in both situations was in some sense unexpected given the increased complexity of the Overhearing condition. First, whereas children in the Addressed condition directly engaged with the experimenter as she introduced novel words, children in the Overhearing condition were positioned as onlookers to such an interaction. Consequently, these children did not have the benefit of establishing mutual eye contact with the experimenter. Instead they had to independently tune in to the adult's focus of attention to determine the referent for the novel word. It is important to note that the target object/ action was not present when the experimenter uttered the novel word; thus, children were not simply passively associating the word with whatever was in sight. Second, compared with children in the Addressed condition, those in the Overhearing condition experienced a greater delay between presentation of the novel word and the comprehension trial. Thus, to demonstrate word learning in the Overhearing condition, children had to retain their knowledge of the appropriate word-referent match for a longer period of time. Finally, children in the Overhearing condition of Study 1B (verb learning) did not have as much time to explore the actions themselves before comprehension testing. Despite these potential difficulties, these children demonstrated an impressive ability to monitor conversations between others and successfully learn novel words from these conversations. 
With younger children, however, the results were somewhat different. In Studies 2 and 3, we examined the abilities of younger 2-year-olds to determine whether they, too, were adept at learning words through overhearing. In Study 2A, these children clearly demonstrated an ability to learn object labels through overhearing. Thus, as with the older 2-yearolds, the younger 2-yearolds were able to overcome the complexities of the Overhearing condition and successfully learn new object labels.

The ability of younger 2-year-old children to learn novel action verbs, however, was not as clear. In Study 2B, conservative statistical analyses (sign tests) did not reveal any indication of novel action verb learning in either the Addressed or Overhearing condition. Binomial tests against chance, however, suggested that children in the Addressed condition did demonstrate verb learning. In Study 3 (with the revised Overhearing procedure), binomial tests revealed that children in both conditions demonstrated verb learning, yet once again results of the sign tests did not provide evidence of verb learning in either condition. Thus, the present studies do not provide unequivocal evidence of the conditions under which younger 2-year-old children are able to learn verbs. It should be noted, however, that the findings for children in the Addressed condition are inconsistent with previous work by Akhtar and Tomasello (1996). Clear evidence was found, however, to support claims that even younger 2-year-old children are able to learn object labels through overhearing.

There are several reasons why learning object labels may be easier than learning verbs for children. Gentner (1982), for example, suggests that the concrete quality and greater cohesiveness of objects, compared with actions, may make objects more accessible semantic targets. Thus, children may be more successful at learning object labels because objects present longer-lasting (stable) percepts, whereas actions are generally more fleeting and more complex in that they represent relations among entities (Gentner, 1978). Another possible explanation for children's greater success at learning object labels compared with learning action verbs may 
relate to the syntactic structure of child-directed speech. Examinations of parents' speech to children have found that, at least in English-speaking, Western middle-income families, parents often make object labels prominent by placing them at the ends of utterances and giving them vocal emphasis (Fernald \& Morikawa, 1993; Messer, 1981; Tardif, Shatz, \& Naigles, 1997). In testing the importance of this typical caregiver speech pattern, Shady and Gerken (1999) found that younger 2-yearold children were more likely to comprehend object labels that were presented in utterance-final position than those presented in utterance-internal position. This result is relevant to our current findings in that, in our language models, object labels were presented in utterance-final position, whereas verbs were internal to the utterance. Although the children, age 2,6, in our studies were able to learn verbs despite their internal position, the younger 2-year-olds did not clearly demonstrate verb learning. As the actions employed were all causative, transitive frames to present the novel verb had to be used. Future research might consider whether younger 2-year-old children would be more successful at verb learning when intransitive frames are used, such that the target verb falls at the end of the language model and receives more stress than it does in transitive sentence frames (e.g., “Look! Tigger’s meeking.”).

Although there are a variety of reasons to expect verb learning to be more difficult than object label learning, it is not neccesarily the case that this pattern is consistent across cultures and linguistic groups. Cross-linguistic observations, for example, suggest that the relative emphasis on nouns versus verbs in caregiver speech may differ across languages. In particular, Korean speakers tend to emphasize verbs to a greater extent than nouns, and Korean children learn to comprehend and produce verb forms earlier than their English-speaking age-mates (Choi \& Gopnik, 1995; Gopnik \& Choi, 1995). Similarly, a sample of young Mandarin-speaking children produced slightly more verbs than nouns, reflecting Mandarin-speaking caregivers' emphasis on verbs over nouns (Tardif, 1996). Further experimental cross-linguistic studies are needed to determine whether children from linguistic backgrounds that emphasize verbs have an easier time learning verbs 
through overhearing than did the younger 2-year-old children in the present studies. Similarly, it is possible that children from cultural communities in which observation (rather than direct teaching) is the predominant mode of learning (Rogoff, Mistry, Göncü, \& Mosier, 1993) might perform better at an earlier age in overhearing situations, due to the extended practice they have had in learning through observation.

Another factor that may have influenced the younger children's performance with novel verbs in these studies was the amount of exposure to the novel actions they had before encountering the verb describing the target action. Children gain nonlinguistic knowledge through repetitive interactions (routines)— often referred to as “event knowledge” (Nelson, 1986)—-that enables them to more easily attach words subsequently encountered in those contexts to their appropriate referents. The general idea is that event knowledge allows children to anticipate objects and actions involved in a given event, and in a sense provides them with a nonlinguistic understanding of the situation that then frees up attention to the accompanying linguistic components of those events. In relation to the current studies, it is possible that for younger two-year-olds, the amount of preexposure to the novel actions (even though it was increased in Study 3) was not sufficient to enable them to focus attention on the language used by the experimenter. Consequently, insufficient event knowledge, along with the other factors discussed above, may have contributed to their difficulty in learning action verbs in Studies 2B and 3.

That children learn words through overhearing calls into question the view that language learning requires joint attention between child and adult. Researchers have demonstrated that children learn words best when adults label objects children are already looking at, rather than objects that require them to shift their attentional focus (Dunham, Dunham, \& Curwin, 1993; Tomasello \& Farrar, 1986). Other studies have shown that young children play an active role in establishing joint attention with caregivers (Akhtar \& Tomasello, 1996; Baldwin, 1991, 1993; Tomasello \& Barton, 1994). For example, upon hearing an adult utter a novel label for one of 
several possible novel objects (e.g., "It’s a toma”), young toddlers will check to see where the adult is looking (Baldwin, 1991). In addition, toddlers also resist word learning when joint attention cannot be established (Baldwin et al., 1996).

These findings all suggest that social cues to a speaker's focus of attention (e.g., gaze direction) facilitate infants' attempts at making word-referent connections. Given the important role that joint attention seems to play in word learning, the current set of findings may seem somewhat controversial. In the present work, with the exception of 2-year-olds learning novel action verbs, children were able to learn novel words equally well when positioned as an onlooker to an interaction between two adults as when interacting directly with an adult. Thus, although one might assume that engaging directly with an adult in establishing a shared focus of attention would be advantageous to word learning, our findings show that children are able to acquire vocabulary outside of such interactions.

Joint attention has been described as the "simultaneous engagement of two or more individuals in mental focus on one and the same external thing” (Baldwin, 1995, p. 132), but also as the “intersubjective awareness that accompanies joint attention, the recognition that mental focus on some external thing is shared” (Baldwin, 1995, p. 132). Most research has focused on the achievement of joint attention in face-to-face interactions. The present findings suggest that the two individuals (speaker and listener) need not be intersubjectively engaged for the listener to be able to learn a word that the speaker utters. In the Addressed conditions of our studies, a shared focus of attention was achieved by child and experimenter as they both took responsibility for checking the others’ line-ofregard. Not surprisingly, by age 2,6, children are very good word learners in this context. In the Overhearing conditions, however, the responsibility for determining the adult's focus fell exclusively on the children because the experimenter was not attending to the children and their attentional focus. By age 2, our participants were able to do so and could reliably learn object labels in such settings; by age 2,6 they learned both object labels and action verbs. 
The overhearing context may occur frequently in children's daily experiences. As children participate in day-to-day activities, they are likely to overhear conversations between others that include unfamiliar words. To make sense of those unfamiliar words, children need to tune in to the speaker's focus of attention. In everyday life, children may have multiple opportunities to overhear a given unfamiliar word and attempt to determine its referent. As discussed earlier, there is evidence that repeated exposure to personal pronouns in an overhearing context fosters learning of these words (Oshima-Takane, 1988, 1999). In particular, Oshima-Takane (1988) found that laterborn siblings have an advantage over firstborns in learning personal pronouns, presumably because they have more opportunities to overhear pronouns being used in conversations between their siblings and parents. Thus, Oshima-Takane’s work shows that repeated experience overhearing personal pronouns is associated with advanced learning of those words. The present studies extend this research in at least two ways: (1) by demonstrating that word learning through overhearing can also be achieved in a single learning episode and (2) by demonstrating that young children can learn other word types (object labels, action verbs) through overhearing. Of course, the experimental situation we employed is likely much simpler than real-life overhearing contexts in that the children were seated in view of the adults and there was little to distract them from attending to the interaction between those adults. It remains to be seen whether 2-yearolds are also able to acquire words through overhearing in more natural and perhaps more challenging settings in which they have multiple events competing for their attention (see Bloom, 2000, on the importance of engagement in children's early word learning).

Although studies that systematically vary the level of surrounding, and potentially distracting, activity need to be conducted, informal conversations with the parents of our participants suggest that children may indeed be capable of learning vocabulary in these more challenging settings. Many parents reported that their children knew many more words than they had been explicitly taught (including some words that parents would prefer their children had not learned!) We do 
not expect the findings of this study to come as a surprise to most parents of young children, nor is it expected that these findings will surprise researchers studying cultural differences in language socialization. Several studies have demonstrated that the amount of time young children spend in face-to-face conversations varies significantly across cultural groups. Thus, although face-toface conversations may be a component of some children's interactions some of the time, there is a wide range of environments in which children learn to talk, and learning through overhearing may play a particularly important role in cultures where children are not often provided with direct instruction (Lieven, 1994).

In summary, the present studies provide direct research evidence in support of the idea that children acquire vocabulary in nondidactic, nonostensive contexts. Specifically, the findings support claims that young children are quite adept at monitoring third-party conversations, and provide experimental evidence that they can acquire vocabulary in such situations. Together with the results of ethnographic studies demonstrating very little direct language teaching in interactions between adults and young children, these results highlight the active role played by children in acquiring language, and point to the possibility that didactic interactions might be the exception rather than the rule in the contexts in which young children in all cultures acquire their early vocabulary.

\section{ACKNOWLEDGMENTS}

The authors would like to thank all the families who participated, and the following students who assisted in data collection and coding: Christian Balducci, Sabina Brown, Kanoelani Connor, Nicole Costello, Antoinette Dasbach-Prisk, Kathrina Firme, Monna Gal-van, Lisa Montague, Vicky Olthof, Cindy Takamoto, and Bria Wrede-Alba. Special thanks go to Margarita Azmitia, Lois Bloom, and an anonymous reviewer for their helpful comments on a previous version of this manuscript.

\section{ADDRESSES AND AFFILIATIONS}

Corresponding author: Nameera Akhtar, Psychology Department, University of California, Santa Cruz, CA 95064; e-mail: nakhtar@cats.ucsc.edu. Jennifer Jipson and Maureen A. Callanan are also at the University of California at Santa Cruz. 


\section{REFERENCES}

Akhtar, N., \& Tomasello, M. (1996). Twenty-four-month-old children learn words for absent objects and actions. British Journal of Developmental Psychology, 14, 79-93.

Akhtar, N., \& Tomasello, M. (1998). Intersubjectivity in early language learning and use. In S. Braten (Ed.), Intersubjective communication and emotion in early ontogeny (pp. 316- 335). New York: Cambridge University Press.

Akhtar, N., \& Tomasello, M. (2000). The social nature of words and word learning. In R. M. Golinkoff \& K. Hirsh-Pasek (Eds.), Becoming a word learner: A debate on lexical acquisition (pp. 115-135). Oxford, U.K.: Oxford University Press.

Baldwin, D. A. (1991). Infants' contribution to the achievement of joint reference. Child Development, 62, 875-890.

Baldwin, D. (1993). Infants' ability to consult the speaker for clues to word reference. Journal of Child Language, 20, 395-418.

Baldwin, D. A. (1995). Understanding the link between joint attention and language. In C. Moore \& P. J. Dunham (Eds.), Joint attention: Its origins and role in development (pp. 131-158). Hillsdale, NJ: Erlbaum.

Baldwin, D. A., Markman, E. M., Bill, B., Desjardins, R. N., Irwin, J. M., \& Tidball, G. (1996). Infants’ reliance on a social criterion for establishing word-object relations. Child Development, 67, 31353153.

Barton, M. E., \& Tomasello, M. (1991). Joint attention and conversation in mother-infant-sibling triads. Child Development, 62, 517-529.

Bloom, L. (1998). Language acquisition in its developmental context. In D. Kuhn \& R. S. Siegler (Eds.), W. Damon (Series Ed.), Handbook of child psychology: Vol. 2. Cognition, perception, and language (pp. 309-370). New York: Wiley.

Bloom, L. (2000). The intentionality model of word learning: How to learn a word, any word. In R. M. Golinkoff \& K. Hirsh-Pasek (Eds.), Becoming a word learner: A debate on lexical acquisition (pp. 19-50). Oxford, U.K.: Oxford University Press.

Brown, P. (in press). Conversational structure and language acquisition: The role of repetition in Tzeltal adult and child speech. Journal of Linguistic Anthropology.

Callanan, M., Akhtar, N., Sussman, L., \& Sabbagh, M. (2001). Learning words in directive and ostensive contexts. Manuscript submitted for publication.

Choi, S., \& Gopnik, A. (1995). Early acquisition of verbs in Korean: A cross-linguistic study. Journal of Child Language, 22, 497-529.

Crago, M. B., Allen, S. E. M., \& Hough-Eyamie, W. P. (1997). Exploring innateness through cultural and linguistic variation. In M. Gopnik (Ed.), The inheritance and innateness of grammars (pp. 7090). New York: Oxford University Press.

Dunham, P. J., Dunham, F., \& Curwin, A. (1993). Joint-attentional states and lexical acquisition at 18 months. Developmental Psychology, 29, 827-831.

Dunn, J., \& Shatz, M. (1989). Becoming a conversationalist despite (or because of) having an older sibling. Child Development, 60, 399-410.

Fernald, A., \& Morikawa, H. (1993). Common themes and cultural variations in Japanese and American mothers' speech to infants. Child Development, 64, 637-656.

Forrester, M. A. (1992). The development of young children's social-cognitive skills. Hillsdale, NJ: Erlbaum.

Forrester, M. A. (1993). Affording social-cognitive skills in young children: The overhearing context. In D. J. Messer \& G. J. Turner (Eds.), Critical influences on child language acquisition and development (pp. 40-61). New York: St. Martin’s Press.

Fox Tree, J. E. (1999). Listening in on monologues and dialogues. Discourse Processes, 27, 35-53.

Gentner, D. (1978). On relational meaning: The acquisition of verb meaning. Child Development, 49, 988-998. 
Gentner, D. (1982). Why nouns are learned before verbs: Linguistic relativity versus natural partitioning. In S. A. Kuczaj (Ed.), Language development: Vol. 2. Language, thought, and culture (pp. 301-334). Hillsdale, NJ: Erlbaum.

Goodman, J. C., McDonough, L., \& Brown, N. B. (1998). The role of semantic context and memory in the acquisition of novel nouns. Child Development, 69, 1330-1344.

Gopnik, A., \& Choi, S. (1995). Names, relational words, and cognitive development in English and Korean speakers: Nouns are not always learned before verbs. In M. Tomasello \& W. E. Merriman (Eds.), Beyond names for things: Young children's acquisition of verbs (pp. 63-80). Hillsdale, NJ: Erlbaum.

Lieven, E. V. M. (1994). Crosslinguistic and crosscultural aspects of language addressed to children. In C. Gallaway \& B. J. Richards (Eds.), Input and interaction in language acquisition (pp. 5673). Cambridge, U.K.: Cambridge University Press.

Messer, D. J. (1981). The identification of names in maternal speech to infants. Journal of Psycholinguistic Research, 10, 69-77.

Nelson, K. (1986). Event knowledge: Structure and function in development. Hillsdale, NJ: Erlbaum.

Oshima-Takane, Y. (1988). Children learn from speech not addressed to them: The case of personal pronouns. Journal of Child Language, 15, 95-108.

Oshima-Takane, Y. (1999). The learning of first- and second-person pronouns in English. In R. Jackendoff, P. Bloom, \& K. Wynn (Eds.), Language, logic, and concept: Essays in memory of John Macnamara (pp. 373-409). Cambridge, MA: MIT Press.

Oshima-Takane, Y., Goodz, E., \& Derevensky, J. L. (1996). Birth-order effects on early language development: Do secondborn children learn from overheard speech? Child Development, 67, 621634.

Reznick, J. S., \& Goldsmith, L. (1989). A multiple form word production checklist for assessing early language. Journal of Child Language, 16, 91-100.

Rogoff, B., Mistry, J., Göncü, A., \& Mosier, C. (1993). Guided participation in cultural activity by toddlers and caregivers. Monographs of the Society for Research in Child Development, 58(7, Serial No. 236).

Saffran, J. R., Newport, E. L., Aslin, R. N., Tunick, R. A., \& Barrueco, S. (1997). Incidental language learning: Listening (and learning) out of the corner of your ear. Psychological Science, 8, 101-105.

Schieffelin, B. B. (1990). The give and take of everyday life: Language socialization of Kaluli children. New York: Cambridge University Press.

Schieffelin, B. B., \& Ochs, E. (1983). A cultural perspective on the transition from prelinguistic to linguistic communication. In R. M. Golinkoff (Ed.), The transition from pre-linguistic to linguistic communication (pp. 115-131). Hillsdale, NJ: Erlbaum.

Shady, M., \& Gerken, L. (1999). Grammatical and caregiver cues in early sentence comprehension. Journal of Child Language, 26, 163-175.

Tardif, T. (1996). Nouns are not always learned before verbs:

Evidence from Mandarin speakers' early vocabularies. Developmental Psychology, 32, 492-504. Tardif, T., Shatz, M., \& Naigles, L. (1997). Caregiver speech and children's use of nouns versus verbs: A comparison of English, Italian, and Mandarin. Journal of Child Language, 24, 535-565.

Tomasello, M., \& Barton, M. E. (1994). Learning words in nonostensive contexts. Developmental Psychology, 30, 639-650.

Tomasello, M., \& Farrar, M. J. (1986). Joint attention and early language. Child Development, 57, 1454-1463.

Tomasello, M., \& Kruger, A. (1992). Acquiring verbs in ostensive and non-ostensive contexts. Journal of Child Language, 19, 311-333.

Zukow-Goldring, P. (1995). Sibling caregiving. In M. H. Bornstein (Ed.), Handbook of parenting: Vol. 3. Status and social conditions of parenting (pp. 177-208). Mahwah, NJ: Erlbaum. 


\begin{tabular}{lcccr}
\hline & & \multicolumn{3}{c}{ Mean Vocabulary Scoresa } \\
\cline { 2 - 5 } Condition & Study 1A & Study 1B & Study 2A & Study 2B \\
\hline Addressed & $96.0(23.0)$ & $93.2(21.8)$ & $65.7(33.5)$ & $65.7(35.0)$ \\
Overhearing & $95.2(25.0)$ & $98.1(25.8)$ & $63.1(39.4)$ & $63.1(38.1)$ \\
\hline
\end{tabular}

Note: Standard deviations are in parentheses. The mean score in the Addressed condition was not significantly different from the mean score in the corresponding Overhearing condition in any of the studies, all $p$ values $>$.05. a Scores are based on Form A of Reznick and Goldsmith (1989).

Table 2 Mean Length of Time between the Comprehension Trial and the Experimenter's Last Utterance Containing the Novel Word in Each Condition

\begin{tabular}{lllll} 
& \multicolumn{4}{c}{ Mean Length of Time (min) } \\
\cline { 2 - 5 } Condition & Study 1A & Study 1B & Study 2A & Study 2B \\
\hline Addressed & $2.49(.84)$ & $1.98(.92)$ & $2.31(.68)$ & $1.88(.59)$ \\
Overhearing & $3.35(1.09)$ & $3.01(.84)$ & $3.53(.99)$ & $2.43(.68)$ \\
\hline
\end{tabular}

Note: Standard deviations are in parentheses. In each study, the delay in the Overhearing condition was significantly longer than in the Addressed condition, all $p$ values $<.05$.

Table 3 Number of Children $(N=12)$ in Study 1 Who Chose the Target Object (or Performed the Target Action) on the Comprehension and Preference Trials

$$
\text { Number of Children }
$$

Study 1A: Object

Study 1B: Action

\begin{tabular}{lcccc}
\cline { 2 - 4 } & Comprehension & Preference & Comprehension & Preference \\
\hline Addressed & $10^{*}$ & 2 & $8^{*}$ & 1 \\
Overhearing & $10^{*}$ & 2 & $7^{*}$ & 0 \\
\hline
\end{tabular}

$* p<.05$ (binomial test).

Table 4 Number of Children $(N=16)$ in Study 2 Who Chose the Target Object (or Performed the Target Action) on the Comprehension and Preference Trials

\begin{tabular}{lcccc} 
& \multicolumn{3}{c}{ Number of Children } \\
\cline { 2 - 4 } & \multicolumn{2}{c}{ Study 2A: Object } & Study 2B: Action \\
\cline { 2 - 5 } Condition & Comprehension & Preference & 4 & Comprehension \\
\hline Addressed & $12^{*}$ & 1 & $8^{*}$ & 4 \\
Overhearing & $9^{*}$ & 4 & 4 \\
\hline
\end{tabular}

$* p<.05$ (binomial test).

Table 5 Number of Children $(N=16)$ in Study 3 Who Chose the Target Object (or Performed the Target Action) on the Comprehension and Preference Trials

Condition

Addressed

Overhearing

${ }^{*} p<.05$ (binomial test).
Number of Children
Comprehension Preference

$\begin{array}{ll}8^{*} & 4 \\ 7^{*} & 2\end{array}$


